Sehr interessant, und mich persönlich am meisten nachdenklich stimmend, fand ich das Abschlusskapitel von Martin Herberhold, der über mögliche Auswirkungen der in den Industrienationen fortschreitenden Digitalisierung auf unsere psychotherapeutische Tätigkeit und das Selbstverständnis von Psychotherapeut/innen nachdenkt und schreibt.

Insgesamt illustriert auch das vorliegende Buch den in der Psychotherapieforschung vielfach publizierten Befund, dass einzelne Psychotherapeut/innen ihre Ausbildungen in einer bestimmten Psychotherapiemethode so lange um weitere Konzepte und Techniken (mehr oder weniger eklektisch) erweitern, bis sie sich den Problemstellungen im klinischen Alltag einigermassen gewachsen fühlen.

Das Buch sei allen, denen bisher der Körper in der Psychoanalyse gefehlt hat, und denen, die in Körperpsychotherapien einen gewissen Mangel an kognitiver Raffinesse vermisst haben, empfohlen.

Margit Koemeda

\section{Frank-M. Staemmler (2017). Relationalität in der Gestalttherapie: Kontakt und Verbundenheit}

\author{
Gevelsberg: EHP. ISBN: 978-3-89797-103-5. \\ 292 Seiten. 26,99 EUR, 41,99 CHF
}

\author{
Psychotherapie-Wissenschaft 9 (1) 86-87 2019 \\ www.psychotherapie-wissenschaft.info \\ CC BY-NC-ND \\ https://doi.org/10.30820/1664-9583-2019-1-86
}

Gleich zu Beginn stellt Frank-M. Staemmler klar, was er mit dieser Neuerscheinung beabsichtigt:

«Ich verstehe den vorliegenden Text eher als ein Zusammenführen und eine Fortsetzung früherer Gedankengänge. Ich knüpfe dabei hauptsächlich an die Inhalte dreier meiner Bücher an: Therapeutische Beziehung und Diagnose - Gestalttherapeutische Antworten (1993), Das Geheimnis des Anderen - Empathie in der Psychotherapie (2009a) und Das dialogische Selbst - Postmodernes Menschenbild und psychotherapeutische Praxis (2015a)» (S. 15).

Der gemeinsame Fokus ist die menschliche Relationalität. Mit Zitaten am Anfang von jedem Kapitel ehrt Staemmler den 1949 verstorbenen Hans Trüb, dessen posthum erschienenes Werk Heilung aus der Begegnung - Überlegungen zu einer dialogischen Psychotherapie einen Grundstein für die Relationalität in der Psychotherapie gelegt hat.

Staemmler folgt der historischen Spur des Paradigmenwechsels von einer "Ein-Person-Psychologie» (bei der Individualismus in den 1960er und 1970er Jahren im Vordergrund stand) zur «Zwei-Personen-Psychologie» und zwischenmenschlichen Wechselseitigkeit der sogenannten relationalen Wende (die 1980er Jahre). Was die Gestalttherapie betrifft, so hat die relationale Wende zwei
Schwerpunkte hervorgebracht. Der eine Schwerpunkt bezieht sich auf ethische Gesichtspunkte, die Gestalttherapeuten und Gestalttherapeutinnen sich zu Herzen nehmen sollen: sich möglichst um eine Ich-Du-Haltung im Sinne Martin Bubers in der therapeutischen Arbeit bemühen und die gemeinsame Suche nach Bedeutung, was die Aufgabe einer unangebrachten Haltung der Unfehlbarkeit seitens des Therapeuten bzw. der Therapeutin impliziert. Der zweite Schwerpunkt ist inhaltlicher Natur: Therapeuten und Therapeutinnen sollten sich nicht nur auf die individuellen psychologischen Prozesse ihrer Patienten und Patientinnen konzentrieren, sondern auch die eigenen Prozesse wie die zwischenmenschlichen Ereignisse, die sich in der Therapiestunde einstellen, sollen volle Aufmerksamkeit erhalten.

Staemmler plädiert dann für einen weiteren Paradigmenwechsel, vom «Selbst-in-Beziehung» zum «relationalen Selbst». "Schwache Relationalität», ein Begriff, den Staemmler von Brent Slife entlehnt, beschreibt Ersteres. «Selbst-in-Beziehung» kann man «als ein Individuum interpretieren, das primär aus sich selbst heraus existiert und dann, in einem zweiten Schritt, auf andere trifft, sich auf Beziehungen mit ihnen ein- und sich dann von ihnen beeinflussen lässt» (S. 175). Letzteres erweitert den Begriff von Relationalität, begreift Menschen als grundsätzlich aufeinander bezogen sowie miteinander verwoben und wird als «starke Relationalität» bezeichnet. Bezugnehmend auf Gestaltkollegen und Gestaltkolleginnen und andere Therapieschulen betont Staemmler wie Beziehungen und dynamische Wechselseitigkeiten Voraussetzungen für die Entwicklung des Selbst und der wahrnehmbaren Welt sind, nicht umgekehrt.

Das Buch ist historisch aufgebaut und Staemmler fügt, wie in früheren Publikationen, «Textboxen» mit zahlreichen Beispielen aus seiner Praxis oder passende theoretische Ergänzungen dem Haupttext hinzu. Die Fallvignetten zeigen einerseits exemplarisch wie Staemmlers Ansatz in der Praxis aussieht, andererseits machen sie deutlich, wie sehr er selbst von der Gegenseitigkeit in seinem Beruf als Psychotherapeut profitiert:
«Denn meine Tätigkeit als Psychotherapeut gestattet mir, einen großen Teil meines beruflichen Lebens in Verbundenheit mit anderen Menschen und in Fürsorge für sie zu verbringen. So sehr es von außen betrach- tet den Anschein haben mag, als engagierte ich mich hauptsächlich für meine Klienten, so sehr tue ich dabei ständig auch viel für mich selbst und für mein eigenes Wohlbefinden. Das Erleben von Resonanz und Verbun- denheit - ungeachtet der jeweiligen Einzig- und Anders- artigkeit meiner Klientinnen - sowie die Haltung der Gastlichkeit und Fürsorge sind dabei neben dem Erwerb von Anerkennung und der Erfahrung von Selbstwirk- samkeit die für mich maßgeblichen Faktoren» (S. 10).

Eine Ermutigung für Praktizierende, jung und alt, in einem Arbeitstag, der mit einer verwirrenden Mischung aus Glück, Betroffenheit und Erschöpfung endet, eine Quelle der Kreativität und Regenerierung zu finden. 
Für mich kommt eines der Herzstücke des Buches in «Anhang 1: Der Wille zur Unsicherheit - Vorläufige Überlegungen über Interpretation und Verstehen in der Gestalttherapie» (S. 241) vor. Dessen Schwerpunkt liegt auf einem Thema, das mir seit vielen Jahren sehr wichtig ist (Amendt-Lyon, 2006a und b): der kreative Vorgang der gemeinsamen Bedeutungsfindung. Hier finden interessierte Leser und Leserinnen Ausführungen zur bereits von Staemmler beschriebenen therapeutischen Haltung der «kultivierten Unsicherheit», zur Unmöglichkeit als Psychotherapeut bzw. Psychotherapeutin nicht zu interpretieren und zum Verstehen als «innovativen Vorgang, in dessen Verlauf neuer Sinn geschaffen wird» (S. 263; Herv. d. V.). So findet eine Erweiterung des eigenen Horizonts statt, damit der Horizont des Anderen inkludiert werden kann. "Wenn Verstehen Teil des Menschenseins ist, ist es Teil unserer Lebendigkeit. Und was ist das Wesen des Lebendigen, wenn nicht seine kontinuierliche Fortpflanzung und Kreativität?» (S. 264).

«Anhang 2: KlientInnen-Fragebogen zur Kontakt und Beziehung» beschäftigt sich mit der Frage, «welche Auswirkungen die Beziehung zwischen KlientInnen und ihren TherapeutInnen auf den therapeutischen Veränderungsprozess hat» (S. 269), und wie Therapeuten und Therapeutinnen nach sehr intensiven Therapiesitzungen durch Klienten und Klientinnen erlebt werden.

Staemmlers Text ist detailreich und seine Literatur umfassend. Umso mehr wundert es mich, dass der Bezug auf John Bowlby und die Bindungstheorie vernachlässigt wird, zumal die bedeutsamen Experimente an Kleinkindern und ihren Müttern in «fremden Situationen» auf Kurt Lewins Forschung bezogen sind. Schade auch, dass die Diskussion der Publikationen von solchen Gestaltkollegen wie Wollants, Francesetti, Gecele und Roubal zur Gestalttherapie als Therapie der gemeinsamen Situation in seinem umfangreichen, sorgfältig recherchierten Werk nicht berücksichtigt wurde. Staemmlers Ausführungen weisen auf eine Ethik des Mitgefühls und der Fürsorge füreinander im Sinne von Lévinas. Nicht nachvollziehbar ist es dann für mich, im selben Band vom «wesenhaft Mitsein» und von der «Bezogenheit von Menschen aufeinander» (S. 173) im Zusammenhang mit Heidegger zu lesen. Erst recht seit der Veröffentlichung seiner «Schwarzen Hefte» ist Heidegger für mich in Bezug auf Menschlichkeit und zwischenmenschliche Beziehungen nicht glaubwürdig, sondern in diesem Kontext deplatziert. Über den Umgang mit Konflikten und destruktiven Kräften in der Relationalität hätte ich gern mehr gelesen.

Zusammenfassend meine ich, dass Staemmler nicht nur nachvollziehbar erklärt, wie sein Verständnis vom relationalen Selbst entstanden ist, sondern seine Beschreibung der Implikationen für die Herstellung und Entwicklung psychotherapeutischer Beziehungen gut gelungen ist. Dieses Buch ist für Gestalttherapeuten und Gestalttherapeutinnen wie auch für alle Therapieschulen eine wertvolle, zeitgemässe Lektüre.

Nancy Amendt-Lyon

\section{Literatur}

Amendt-Lyon, N. (2006a). Auf dem Weg zu einem gestalttherapeutischen Konzept zur Förderung des schöpferischen Prozesses. In M. Spagnuolo Lobb \& N. Amendt-Lyon (Hrsg.), Die Kunst der Gestaltherapie. Eine schöpferische Wechselbeziehung (S. 7-25). Wien, New York: Springer.

Amendt-Lyon, N. (2006b). Denkwürdige Augenblicke in der therapeutischen Beziehung. In M. Spagnuolo Lobb \& N. AmendtLyon (Hrsg.), Die Kunst der Gestalttherapie. Eine schöpferische Wechselbeziehung (S. 241-255). Wien, New York: Springer.

Bowlby, J. (1989 [1979]). The Making \& Breaking of Affectional Bonds. London: Tavistock/Routledge.

Francesetti, G., Gecele, M. \& Roubal, J. (Hrsg.). (2016). Gestalttherapie in der klinischen Praxis. Von der Psychopathologie zur Ästhetik des Kontakts. Gevelsberg: EHP.

Trüb, H. (2015 [1951]). Heilung aus der Begegnung - Überlegungen zu einer dialogischen Psychotherapie. Bergisch Gladbach: EHP.

Wollants, G. (2012 [2008]). Gestalt therapy. Therapy of the Situation. London: Sage.

\section{Nancy Amendt-Lyon (Hrsg.). (2017). Zeitlose Erfahrung. Laura Perls' unveröffentlichte Notizbücher und literarische Texte 1946-1985}

\author{
Gießen: Psychosozial-Verlag. \\ ISBN: 978-3-8379-2702-3. 354 Seiten. \\ 39,90 EUR, $43,90 \mathrm{CHF}$
}

Psychotherapie-Wissenschaft 9 (1) 87-88 2019

www.psychotherapie-wissenschaft.info

CC BY-NC-ND

https://doi.org/10.30820/1664-9583-2019-1-87

In diesem Buch werden bisher unveröffentlichte Texte, Briefe, Gedichte sowie Übersetzungen von Gedichten, Kurzgeschichten und Entwürfe für Vorträge oder Publikationen aus den Tagebuchnotizen von Laura Perls publiziert.

Laura Perls, geborene Lore Posner aus Pforzheim, war die Gattin von Fritz Perls. Seit Jahrzehnten ist bekannt, dass sie eine wesentliche Rolle in der Konzeption und Entwicklung der Gestalttherapie spielte. Ihrem seinerzeitigen Frauenbild entsprechend, wirkte sie jedoch im Hintergrund und überliess die Bühne für Fachpublikationen ihrem Mann und anderen Kollegen. Unter eigenem Namen veröffentlichte sie nur wenig.

Es war die Tochter des Ehepaares Perls, Renate Perls, die die Herausgeberin anfragte, ob sie aus dem Nachlass dieser Tagebücher nicht eine Publikation machen könnte. Eine Aufgabe, die die Wiener Gestalttherapeutin mit amerikanischen Wurzeln Nancy Amendt-Lyon gerne annahm und hervorragend meisterte. Die hinterlassenen Materialien sind zum Teil sehr fragmentarisch und wurden von der Herausgeberein sehr sorgfältig und umsichtig editiert und kommentiert.

Das Buch erschien 2016 erst auf Englisch unter dem Titel Timeless Experience: Laura Perls' Unpublished Notebooks and Literary Texts 1946-1985 im Verlag Cambridge Scholar Publishing, Newcastle. 